\title{
Fetomaternal outcomes in premature rupture of membranes at term: a case control study
}

\author{
Suprajna Shetty*, Harish Shetty
}

Department of Obstetrics and Gynecology, K. S. Hegde Medical Academy, Mangalore, Karnataka, India

Received: 26 May 2017

Revised: 05 January 2018

Accepted: 09 January 2018

\section{*Correspondence:}

Dr. Suprajna Shetty,

E-mail: suprajna@gmail.com

Copyright: () the author(s), publisher and licensee Medip Academy. This is an open-access article distributed under the terms of the Creative Commons Attribution Non-Commercial License, which permits unrestricted non-commercial use, distribution, and reproduction in any medium, provided the original work is properly cited.

\section{ABSTRACT}

Background: Premature rupture of membranes is defined as spontaneous rupture of fetal membranes beyond 28 weeks of pregnancy but before the onset of uterine contractions. It occurs in approximately $10 \%$ of all pregnancies and in $70 \%$ it occurs at term. If PROM occurs before 37 completed weeks, it is referred as preterm premature rupture of membranes(PPROM). PROM is associated with adverse outcomes in both, hence its management becomes crucial. It complicates $8 \%$ of pregnancies. Objective of present study was to investigate the labour, maternal and perinatal outcomes.

Methods: 75 patients who entered labour room at term with PROM were taken as cases and those with intact membranes as controls. Investigations are sent, and prophylactic antibiotics were given. Progress of labour, PROM delivery interval, method of induction, mode of delivery along with maternal and fetal outcomes, total duration of hospital stay was noted and compared with controls.

Results: Rate of caesarean is higher in study group (14.7\%) and failure to progress was the common indication. Intrapartum morbidity was higher in the study group (16\%) and perinatal morbidity was $20 \%$. NICU admission and the need for antibiotics were higher in study group and hence longer duration of hospital stay.

Conclusions: PROM is associated with increased incidence of neonatal sepsis and subsequently antibiotics given were higher in cases. There is a higher incidence of caesarean rates and hospital stay. Proper screening of high risk cases and appropriate management is suggested to reduce perinatal morbidity.

Keywords: Chorioamnionitis, Maternal and perinatal morbidity, PROM

\section{INTRODUCTION}

Premature rupture of membranes is an enigmatic condition associated with high risk of maternal and perinatal morbidity and mortality and has management strategies that are often diverse and controversial. Premature rupture of membranes is defined as spontaneous rupture of fetal membranes beyond 28 weeks of pregnancy but before the onset of uterine contractions. ${ }^{1}$ It occurs in approximately $10 \%$ of all pregnancies and in $70 \%$ of the cases it occurs in pregnancies at term. ${ }^{1,2}$ Premature rupture of membranes results from accelerated membrane weakening by various factors through an increase in local cytokines and an imbalance between MMPs and TIMPs, increased protease and collagenase activity and factors that cause increased intrauterine pressure. ${ }^{3}$ Although vaginal GBS colonization does not appear to be associated with PROM, GBS bacteruria has been associated with preterm PROM and low birth weight infants. ${ }^{4,5}$

PROM at term is defined as the leakage of amniotic fluid beginning atleast 1 hour prior to onset of labour $>37$ weeks of gestational age. Approximately $80 \%$ of PROM 
at term begin labour within 24 hours and $95 \%$ within 72 hours. ${ }^{2}$ In a study conducted by Swati Pandey the incidence was reported at $7.71 \% .^{6}$ The incidence of PROM was found to rise 4.4 fold in women undergoing routine pelvic examination in the third trimester by a study by Jiwane. $^{7}$ Act of coitus in the last trimester showed a rise of 6 fold in PROM in a study by Kodkany and Telang. ${ }^{8}$

Shorter the gestational period, longer the latent period. ${ }^{2}$ When $>24$ hours have elapsed before labour ensues it is called prolonged PROM. Prolongation of latency $>24$ hours is associated with increased incidence of chorioamnionitis and neonatal sepsis. ${ }^{9}$

PROM is an unpredictable event and it occurs suddenly. The exact mechanism for PROM is unknown but studies suggest that a variety of mechanical and biochemical pathways are involved which results in weakness and rupture of the chorioamnion. There are a various risk factors associated with PROM namely low socioeconomic status, smoking, genital tract infection, increased intrauterine pressure, incompetent cervix and others. $^{2}$

PROM is associated with increased risk of chorioamnionitis, unfavourable cervix and dysfunctional labour, increased caesarean rates, postpartum haemorrhage and endometritis in mother. In the fetus there is increased occurrence of sepsis, cord prolapse, fetal distress due to increased fetal wastage. Thus, earlier the gestational age at the time of PROM longer is the latency and more the complications.

Management of PROM remains controversial and challenging. Controversy surrounds the role of antibiotics, induction and expectant management. It complicates approximately $8 \%$ of term pregnancies. ${ }^{10}$

Risk of complications is reduced in PROM when antibiotic prophylaxis is used. ${ }^{11}$ The use of antibiotics in PROM at term has been addressed in a Cochrane review in 2002 which concluded that routine antibiotics for term PROM reduce maternal infectious morbidity but have no neonatal benefit. ${ }^{12}$

To avoid complications labour is usually induced once PROM is confirmed. Induction of labour in a patient with unfavourable cervix still remains a challenge. Different methods of induction exist, of which prostaglandins are renowned for cervical ripening and myometrial stimulation. However, there remains the risk of increased caesarean section due to either failure of induction or hyperstimulation. ${ }^{12}$

A careful consideration of various factors and individualization of cases is necessary for appropriate management. So the present study is to analyze the maternal and perinatal outcome in premature rupture of membranes at term.

\section{METHODS}

This was a prospective case control study conducted on 150 patients who entered labour room of K S Hegde Charitable Hospital, Mangalore with history of leaking $\mathrm{P} / \mathrm{V}$ as cases and patients with intact membranes were taken as their controls. All women are counselled about the study and informed written consent is obtained.

\section{Inclusion criteria}

Gestational age > 37 weeks confirmed by dates, clinical examination and ultrasound.

- Lack of uterine contractions for atleast 1 hour from PROM

- Cervical dilatation $<3 \mathrm{cms}$

- Single live pregnancy in vertex presentation

- $\quad$ PROM confirmed by

- Direct visualization

- Fern test whenever required.

\section{Exclusion criteria}

- Gestational age <37 weeks

- Women in labour or uterine contractions $<1$ hour of rupture of membranes

- Cervical dilatation $>3 \mathrm{cms}$

- Previous caesarean section

- Malpresentation/multiple gestation

- Meconium stained liquor

- Contracted pelvis/Cephalopelvic disproportion.

A detailed history was taken, gestational age was confirmed, and general, systemic and obstetric examination was done. All cases and controls were having normal cardiotocography (CTG) findings at the time of admission to labour room and maternal vitals were recorded 4th hourly.

A sterile speculum examination was done, and the condition of vagina and cervix noted. Liquor draining from the OS was observed. The colour and smell of fluid was noted. If no fluid was seen, the patient was asked to cough, and drainage of fluid was looked for. The specimen was collected and subjected to nitrazine test or Fern test. Cervical swab was taken and sent for culture sensitivity.

A single pelvic examination was done to note the Bishop's score, adequacy of pelvis, assessment of CPD and to rule out cord prolapse. Culture and sensitivity of the fluid and blood investigations are sent. Progress of labour along with maternal and fetal condition was monitored. Prophylactic antibiotics were given to study groups. Depending upon the Bishop's score, labour was induced with prostaglandins or accelerated with oxytocin. Time of induction was noted. The labour of each case was closely monitored. 
PROM delivery interval, method of induction and mode of delivery were noted and compared with controls.

Soon after delivery, Apgar score at 1 and 5 minutes, birth weight, sex, congenital anomalies, immediate complications and birth injuries, birth asphyxia, meconium aspiration, sepsis and other associated complications were noted. CRP was done in all cases and controls. Blood culture and sensitivity were taken in cases with PROM >12hours or those with signs/symptoms suggestive of early neonatal sepsis such as inability to suck, lethargy, vomiting even with lesser hours of PROM. The babies were followed up in the postnatal period and the neonatal morbidity and mortality were noted.

Mothers were watched for third stage complications like $\mathrm{PPH}$ and retained placenta. They were followed up in the puerperal period and the caesarean or episiotomy wound were observed for any gape or infection, urinary or respiratory tract infection.

The total duration of hospital stay was also compared in both the groups.

\section{Statistical analysis}

Data were expressed as the mean \pm standard deviation or rate $(\%)$ and were tested for significance using the Student's t-test and chi-square test, Fisher's exact test.

A P value less than 0.05 was considered statistically significant.

\section{RESULTS}

During the 2 year study period 150 patients were enrolled in the study, 75 in the study group and 75 in the control group after fulfilling the inclusion criteria.

Table 1: Maternal demographic.

\begin{tabular}{|c|c|c|c|c|}
\hline \multicolumn{2}{|c|}{ Characteristics } & $\begin{array}{l}\text { Cases } \\
(\mathbf{N}=75)\end{array}$ & $\begin{array}{l}\text { Controls } \\
(\mathrm{N}=75)\end{array}$ & $\begin{array}{l}P \\
\text { value }\end{array}$ \\
\hline \multicolumn{2}{|l|}{ Age } & $27.0 \pm 4.2$ & $26.6 \pm 3.8$ & 0.06 \\
\hline \multicolumn{2}{|c|}{ Gestational age } & $39.1 \pm 1$ & $39.3 \pm 1$ & 0.19 \\
\hline \multicolumn{2}{|c|}{ BMI } & 26 & 27 & 0.30 \\
\hline \multirow{2}{*}{ Parity } & Primi & 50 & 45 & \multirow{2}{*}{0.62} \\
\hline & Multi & 25 & 30 & \\
\hline
\end{tabular}

Characteristics of the women in both the groups are shown in Table 1. There was no significant difference between the groups.

On admission patients with leaking presented with a lower bishop score than controls (Figure 1). Maximum number of patients in control group $(66.7 \%)$ progress and deliver without any interventions when compared to PROM group (13.3\%). Patients taken as control required augmentation with oxytocin $(33.3 \%)$ and no induction with prostaglandins.

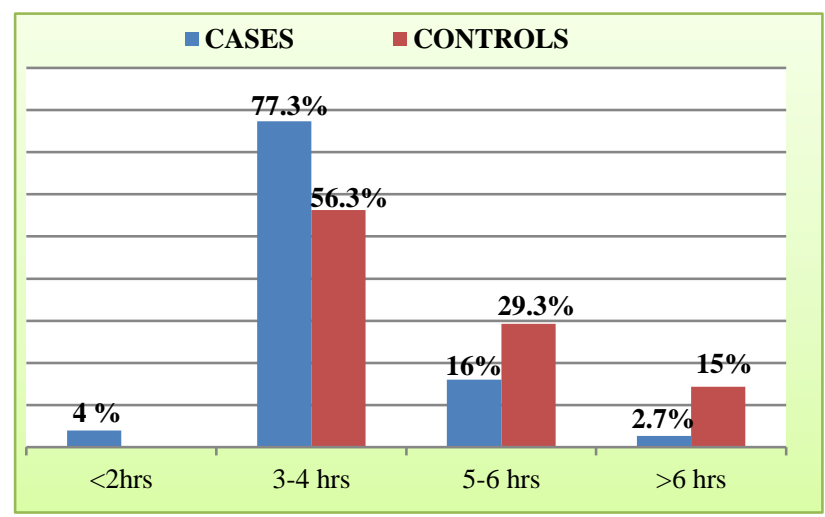

Figure 1: Comparison of Bishop's score.

Most patients with leaking progressed within 6 hours to have a favourable bishop score to be induced with oxytocin and only $18.6 \%$ and $5.4 \%$ patients required induction with PG E2 and PG E1 respectively (Table 2).

Table 2: Comparison between Induction methods.

\begin{tabular}{|lll|}
\hline Induction & Cases & Controls \\
\hline Dinoprostone (1) (PG E2) & $9(12 \%)$ & 0 \\
\hline Oxytocin (2) & $47(62.7 \%)$ & $25(33.3 \%)$ \\
\hline Misoprostol (3) (PG E1) & $2(2.7 \%)$ & 0 \\
\hline Nil & $10(13.3 \%)$ & 0 \\
\hline $\begin{array}{l}\text { Dinoprostone+ } \\
\text { Oxytocin (1+2) }\end{array}$ & $5(6.6 \%)$ & 0 \\
\hline $\begin{array}{l}\text { Misoprostol+ } \\
\text { Oxytocin (3+2) }\end{array}$ & $2(2.7 \%)$ & 0 \\
\hline
\end{tabular}

Mean duration of induction to delivery interval was 5.2 hours \pm 3.2 hours. According to Fishers test $\mathrm{P}$ value is significant for labour outcome at 0.029 which indicates that controls had a higher rate of normal deliveries when compared to PROM. Rate of caesarean section is higher in the study group (14.7\%) unlike those with intact membranes $(2.7 \%)$. Failure to progress was the common indication in both the groups. Instrumental delivery was seen in $5.3 \%$ and $4 \%$ patients with PROM and controls respectively (Table 3 ).

Table 3: Comparison of mode of delivery.

\begin{tabular}{|lllllll|}
\hline & Naginal & & & & \\
& Normal & IVD & Total & & Total \\
\hline Cases & 60 & 4 & 64 & 11 & 75 \\
\hline Controls & 70 & 3 & 73 & 2 & 75 \\
\hline Total & 130 & 7 & 137 & 13 & 150 \\
\hline
\end{tabular}

The delivery interval between cases and controls from admission is highly significant with a mean of $10.8 \pm 3.8$ hours in patients presenting with PROM and 8.9 \pm 2.6 hours in controls. By Mann Whitney t-test $\mathrm{P}$ value is 0.003 which is highly significant. Intrapartum morbidity 
was higher in the study group (16\%) and none of the controls had any intrapartum complications, hence $\mathrm{P}$ value is highly significant at 0.001 (Figure 2).

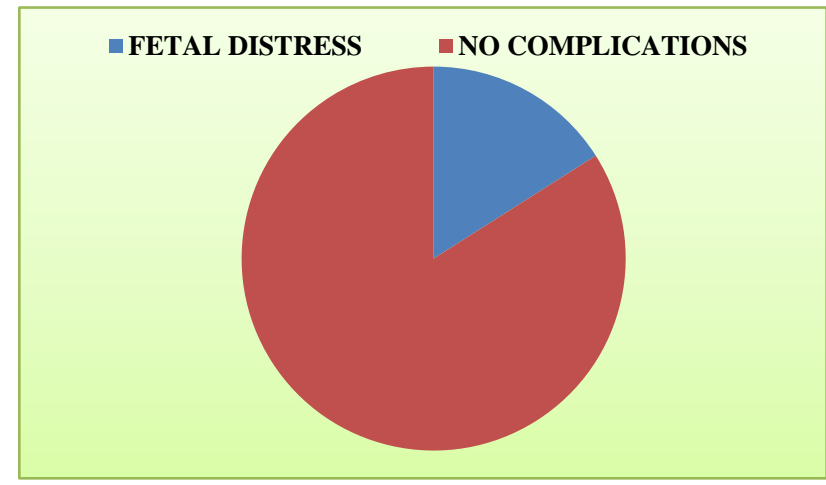

Figure 2: Intrapartum complication in PROM.

Maternal morbidity was seen in several patients in both the groups. Using Chi-square test a $\mathrm{P}$ value of 0.405 was got which is not significant (Table 4).

Table 4: Maternal complications.

\begin{tabular}{|lllll|}
\hline Outcome & Cases & & & \\
& Spontaneous & Induced & Total & Control \\
\hline Fever & 0 & 3 & 3 & 0 \\
\hline TC & 3 & 14 & 17 & 18 \\
\hline CRP +ve & 5 & 20 & 25 & 26 \\
\hline Swab C/S & 0 & 8 & 8 & 4 \\
\hline $\begin{array}{l}\text { Wound } \\
\text { gape }\end{array}$ & 0 & 0 & 0 & 1 \\
\hline PPH & 0 & 0 & 0 & 1 \\
\hline
\end{tabular}

Perinatal morbidity was observed to be $20 \%$ in PROM group and $2.7 \%$ in controls with a $\mathrm{P}$ value of 0.001 which is highly significant (Table 5).

Table 5: Perinatal outcome.

\begin{tabular}{|l|llll|}
\hline Outcomes & Cases & & & \\
& Spontaneous & Induced & Total & Control \\
\hline TC & 0 & 9 & 9 & 3 \\
\hline CRP +ve & 1 & 8 & 9 & 0 \\
\hline Blood C/S & 0 & 1 & 1 & 1 \\
\hline Antibiotic & 1 & 9 & 10 & 3 \\
\hline
\end{tabular}

Respiratory distress was the common cause. NICU admission and the need for antibiotics were higher in the study group due to sepsis. Out of the 75 patients who presented with PROM $25.3 \%$ babies had NICU admissions and in patients taken as control only $5.3 \%$ had NICU admission. $\mathrm{P}$ value is 0.001 which is significant (Figure 3).

Cord CRP was positive only in patients with PROM $(12 \%)$ and there were no positive results in patients taken as controls. $\mathrm{P}$ value $=0.002$ which is highly significant. Vaginal swab culture was positive in $10.7 \%$ of patients with PROM and $5.3 \%$ in controls. $\mathrm{P}$ value $=0.229$, not significant. There was a longer duration of hospital stay in patients with PROM when compared to controls.

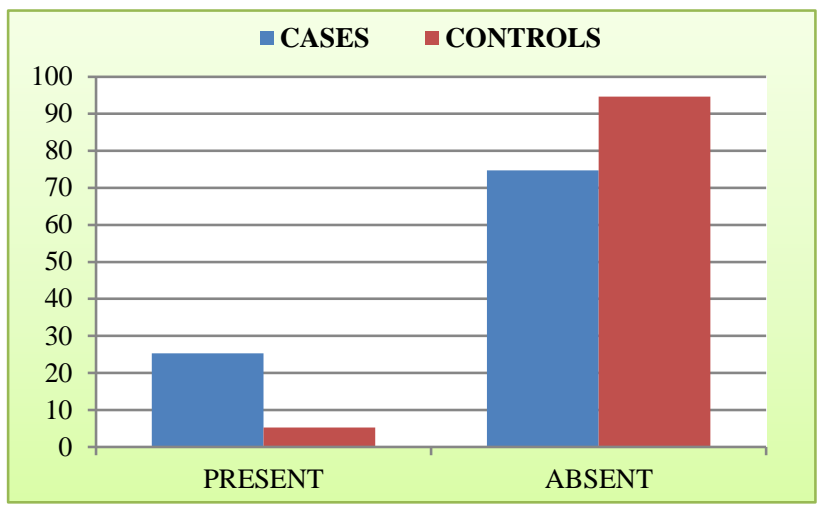

Figure 3: NICU admissions.

The duration of hospital stay can be attributed to the mode of delivery, presence of sepsis in both mother and fetus, use of antibiotics in fetus. In patients with PROM $45.3 \%$ have an average hospital stay of 4-6 days whereas maximum number of patients in control group $(50.7 \%)$ have an average stay within 3 days with a $\mathrm{P}$ value of 0.001 (Figure 4).

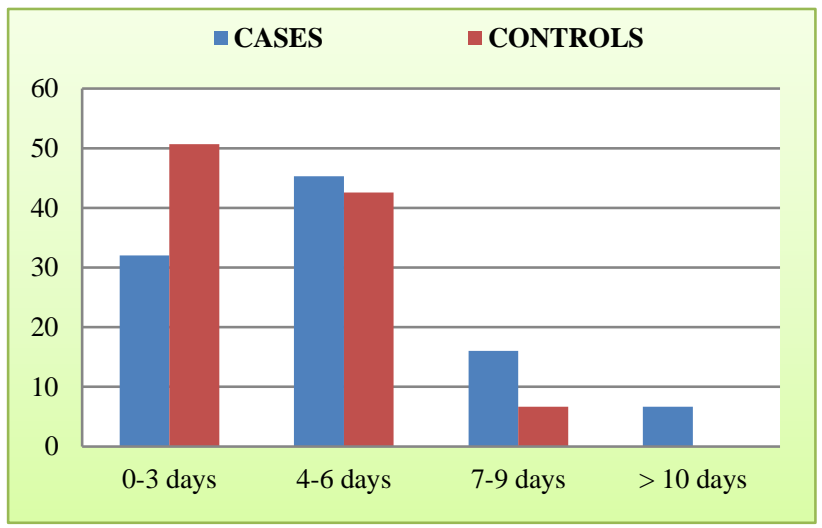

Figure 4: Duration of hospital stay.

\section{DISCUSSION}

Obstetricians from ancient times were of the belief that PROM causes maternal morbidity, increased operative delivery and neonatal morbidity and mortality. To increase obstetrician's difficulty is the fact that the literatures available pertain to the studies in developed countries where there is better facility for neonatal care, strict asepsis is followed, and antibiotics are initiated when necessary. ${ }^{13}$

In developing countries like India there is a higher incidence of perinatal morbidity due to the poor resource setting. Hence use of proper asepsis, antibiotics and induction protocol become necessary to decrease the morbidity. 
PROM is an enigmatic condition complicating 5-10\% of all pregnancies. Diagnosis and management of PROM is complex.

In our study PROM group had a mean age of $27 \pm 4.2$ and controls a mean of $26.6 \pm 3.8$ which is comparable to a study by $\mathrm{Xia} \mathrm{H}$ et al. According to various studies, with increasing maternal age the risk of PROM decreases. ${ }^{14}$

The duration of PROM to admission is important as the morbidity increases with time. Our study showed a maximum duration of 8 hours and the minimum period of 15 minutes. The mean duration being 2.2 hours which is very less compared to other studies like Thakor $\mathrm{U}$ and Devi A where the mean duration was $12.06 \pm 6.04$ hours and 16 hours respectively. ${ }^{15,16}$

In the present study we observed that the rate of normal deliveries was higher in control group (93.3\%) when compared to the PROM group (80\%). Pandey S showed rate of caesarean section in the study group to be $31 \%$ and $12 \%$ in the control group. ${ }^{6}$ Study by Hexia Xia et al concluded that $43.5 \%$ had vaginal delivery, $1.4 \%$ instrumental delivery, $55.1 \%$ caesarean delivery in the PROM group and in controls $55.9 \%, 1.7 \%, 42.5 \% .^{15}$ Present study observed that vaginal delivery comprised of $80 \%, 14.7 \%$ caesarean and $5.3 \%$ instrumental delivery in PROM whereas in controls it constituted 93.3\%, 2.7\% and $4 \%$ respectively. The most common indications for caesarean seen are fetal distress and failure to progress.

In a study by Shah $\mathrm{M}$ et al, $79.2 \%$ cases delivered within 24 hours of PROM. In the present study $76 \%$ cases delivered within 12 hours of PROM $(\mathrm{P}=0.003) .{ }^{14}$

Maternal morbidity was seen similar to several studies by Hexia Xia et al, Devi A (22\%), Kodkany (21\%).The most common maternal morbidity observed in PROM study group was pyrexia., $8,15,16$

Intrapartum complications were seen in PROM group and none in the control group.

Perinatal morbidity increases with the increase in PROM to delivery interval. In a study by $\mathrm{M}$ Shah it was observed to be $32.2 \%$ in cases and $3.8 \%$ in controls whereas our study showed an incidence of $20 \%$ in cases and $2.7 \%$ in controls which is comparable to the other study. Respiratory distress and sepsis were the commonest causes for perinatal morbidity in the study group. Perinatal mortality was not observed in our study.

NICU admissions were significant in cases due to sepsis. The number of babies requiring antibiotics was also significant in case group when compared to controls.

The interval from admission to delivery was longer in the PROM group than in controls. The duration of hospital stay was longer in the case group with an average of 5 days and in controls it was 3 days. In a study by Shah M et al hospital stay was significantly higher in the study group (5.98) when compared to controls (3.96) which is similar to present study. ${ }^{14}$

From this study we conclude that the rate of intrapartum and perinatal morbidity is higher in women presenting with PROM and there is a higher chance of caesarean section rates. Higher number of neonates requires NICU admission and interventions with antibiotics in the study group. The duration of hospital stay is also longer in the study group.

Hence precautions need to be taken to reduce the morbidity and each case should be individualized and managed accordingly.

The limitations of this study are

As we had given prophylactic antibiotics to all patients presenting with PROM the rate of incidence of chorioamnionitis is much lower when compared to other studies.

As per my study, in the PROM group expectant management was observed only for 6 hours as compared to the other studies of 12 hours. Hence the progress of labour and mode of delivery may differ.

Funding: No funding sources

Conflict of interest: None declared

Ethical approval: The study was approved by the Institutional Ethics Committee

\section{REFERENCES}

1. James DK, Steer PJ, Weiner CP. High risk pregnancy management options. $4^{\text {th }}$ ed. Saunders;2010:1091-1100.

2. Arias F, Daftary SN, Bhinde AG. Premature rupture of membranes in practical guide to high risk pregnancy and delivery $3^{\text {rd }}$ ed. Elsevier Health Sciences;2012:240-60.

3. Gabbe S, Niebyl J, Simpson J. Obstetrics: normal and problem pregnancies $6^{\text {th }}$ edition. Elsevier; 2012;713:732.

4. Romero R, Mazor M, Oyarzun E. Is there an association between colonization with group B Streptococcus and prematurity? J Reprod Med. 1989;34:797.

5. Regan JA, Klebanoff MA, Nugent RP. Colonization with group B Streptococci in pregnancy and adverse outsome. VIP study group. Am J Obstet Gynecol. 1996;174:1354.

6. Pandey S, Dave A, Bandi S. Maternal and fetal outcome in cases of prom. J Obstet Gynecol India. 2000;50:63.

7. Jiwane KA. Antenatal vaginal examination as a cause of PROM. J Obstet Gynaecol India. 1991;41:337. 
8. Kodkany, Telang. Premature rupture of membranes, a study of 100 cases. J Obstet Gynecol India 1991;41:492.

9. John G, Keirse Marc JNC. Prelabour rupture of membranes at term. Effective case in pregnancy and child birth. Oxford University Press;1989:1112-7.

10. Premature rupture of membranes. Practice Bulletin No 80. American College of Obstetricians and Gynecologists. Obstet Gynecol. 2007;109:1007-19.

11. Flenady V, King J. Antibiotics for PROM at term. Cochrane Database Syst Rev. 2002;(3):CD001807.

12. Krupa S, Haresh D. Premature rupture of membrane at term: early induction versus expectant management. J Obstet Gynecol India. 2012;62(2):172-5.
13. Shah M, Sandesara P. Fetomaternal outcome in cases of PROM. Gujarat Med J. 2011;66(1).

14. Piya R, Sikdar K, Das A, Ghosh T. Study of cases of chorioamniotics followed premature rupture of membranes. J Obstet Gynaecol India. 1997;47:136.

15. Umed T, Aitra N, Baxi S, Hazra M. Labor characteristics in pre-labor rupture of membranes. J Obstet Gynecology India 1994;44:527.

16. Devi A, Rani R. Premature rupture of membranes: a clinical study. J Obstet Gynaecol India 1996;46:63.

Cite this article as: Shetty S, Shetty H. Fetomaternal outcomes in premature rupture of membranes at term: a case control study. Int J Reprod Contracept Obstet Gynecol 2018;7:725-30. 